\title{
Quality of Service (QoS) of a ZigBee Network through Device Priority with Packet Distribution Technique
}

\author{
Md. Anayt Rabbi, Mohammad Samiul Islam, Anjuman Luna, Mohammad Mobarak Hossain, \\ Nishita Aktar, Shamsun Nahar, Nadira Islam, Md. Faizul Huq Arif
}

Conceptual-ZigBee is a far flung innovation depending on IEEE 802.15.4 convention. This conference is deliberate so that you can understand the physical and multiple get admission to manipulate (MAC) layers for a very low facts charge personal location network (PAN). on this exam we studies the wi-fi of issuer $(Q o S)$ parameters of a ZigBee put together. in this paper a ZigBee far off sensor gadget is set up with each versatile wi-fiand wiwireless ZigBee gadgets. Likewise the dependent tool is accomplished relying on hub need. At that element, the presentation of the planned tool is broke down for three unique parcel conveyance strategies: Poisson, Chi-rectangular and Uniform. The measurements implemented for QoS execution assessment include statistics dropped price, MAC remove, start to completewireless cast off, information wi-fic despatched, information wi-fi were given, and throughput. The critical purpose of this examination is to gauge the effect of hub want and sort of parcel flow techniques in a half of of and half of ZigBee tool.

Watchwords-ZigBee prepare, wi-fi Sensor community (WSN), Riverbed, incredible of service $(Q o S)$, Node priority

\section{CREATION}

a long way off Sensor community (WSN) and wireless personal vicinity network (WPAN) is growing wireless greater famous to the scientists in slight of its unique uses of checking, controlling, and computerization of home or places of work. ZigBee is an innovation of open, international some distance off tool favored that is one of the key empowering advances for the wi-fi of every WSN and WPAN. It has low energy vivaciously supposition minimum strive, high growth throughput [1]. ZigBee is furthermore meant for wi-fi station control framework, for example, stressful framework, lights

Revised Version Manuscript Received on 10, September 2019.

Md. Anayt Rabbi, Department of Computer Science and Engineering (CSE), World University of Bangladesh, South Asia.

Mohammad Samiul Islam, Department of Computer Science and Engineering (CSE), World University of Bangladesh, South Asia.

Anjuman Luna, Department of Computer Science and Engineering (CSE), World University of Bangladesh, South Asia..

Mohammad Mobarak Hossain, PhD Fellow, Department of Computer Science and Engineering (CSE),Dhaka University of Engineering and Technology (DUET), Bangladesh, South Asia.(Email: mobarak3112@gmail.com)

Mst. Nishita Aktar, Department of Computer Science and Engineering (CSE), World University of Bangladesh, South Asia.

Shamsun Nahar, Department of Computer Science and Engineering (CSE), World University of Bangladesh, South Asia..

Nadira Islam Department of Computer Science and Engineering (CSE), World University of Bangladesh, South Asia..

Md. Faizul Huq Arif, Assistant Programmer, Department of Information and Communication Technology (DoICT), Ministry of Posts, Telecommunications \& Information Technology, Bangladesh,South Asia.

(Email: arifict27@gmail.com) framework internet of things (IoT) person, domestic and emergency sanatorium care, telecom software program industrial enterprise business enterprise wi-fi mechanization thus on[2][3]. ZigBee Alliance proposed ZigBee convention with the utility layer, network layer, Medium get access to control (MAC) and physical layer. The Institute of electrical and Electronics Engineers (IEEE) institutionalized it is MAC and physical layer as IEEE 802.15.4. [3]. It has moreover one-of-a-kind range enactment for human software. ZigBee is more wi-fi than wi-fi or Bluetooth for little energy. It has the high-quality gabbing of crude of ultra-modern like compact gadgets. It is going fact that the Bluetooth does no longer have introduced collectively data circulate cognizance. Bluetooth and wireless provide brief-pass correspondence and characteristic the network as much as a hundred meters. similarly, wireless and Bluetooth are immoderate statistics charge fashions which bolster the change of media statistics, programming, and device to device (D2D) correspondence loosened up execution and so on [4][5].

ZigBee WSN bolsters records skip charge among 20kbps ( $868 \mathrm{MHz})$ to $250 \mathrm{kbps}(2.4 \mathrm{GHz}$ band). It actually works below the IEEE 802.15.4 preferred physical radio recurrence self-discipline. ZigBee has unlicensed radio recurrence band, at fantastic frequencies together with $868 \mathrm{MHz}, 902-968$ $\mathrm{MHz}$, and multiple. $4 \mathrm{GHz}$. considering the truth that ZigBee calls for low strength usage from the tool, the battery existence is essentially advanced and lengthy lifestyles execution [6].

A ZigBee device includes of 3 kinds of gadgets - ZigBee facilitator, ZigBee switch, and ZigBee stop tool. A ZigBee prevent device can be a savvy CCTV digital digital camera, indoor regulator, tv, and entryway, and so on. In a ZigBee prepare there need to be in any occasion one organizer as it's miles going approximately as a scaffold and root for the whole system [7]. In a powerful ZigBee set up the quantity of ZigBee organizers, ZigBee switches, and ZigBee quit gadgets is based upon the shape of topology actualized in the tool, as an instance, work topology, superstar topology, and tree steerage topology. In wireless gadget, ZigBee facilitator can disguise sixty five,535 gadgets. The cease devices of a ZigBee gadget are set at various spot. wi-fi, inside the full-size scope of ZigBee arrange some of ZigBee gadgets are interconnected. 


\section{QUALITY OF SERVICE (QOS) OF A ZIGBEE NETWORK THROUGH DEVICE PRIORITY WITH PACKET DISTRIBUTION TECHNIQUE}

For the installed order of any gadget, execution is the critical truth. inside the area of media transmission and structures management of any processing device wi-notable of carrier (QoS) is characterised through a few device parameters like throughput, put off, bundle deal deal misfortune percent, bit blunder rate, switch velocity skillability, jitter, lining deferral, and crash likelihood [8].

In home and wi-fi prepare, possible coordination is wanted. For better execution there want a mixture of portable and stuckwireless ZigBee devices. For an full-size tool, a large degree of wi-fi coping with and quit gadgets desires synchronization is needed. As a manner to accumulate better wiwireless of carrier (QoS), need booking is a promising element [1].

in this art work, we research a circulate breed course of movement of ZigBee set up with the aid of creating one in each of a type conditions. if you want to accomplish gifted tool detailing need detail of the object [1] is applied in this planned system. considering electricity sparing in some unspecified time in the future of rest time, bunch tree topology is picked for this gadget.

\section{STUDIES TECHNIQUE}

as a way to wi-fi the development of wi-fiwireless Sensor network (WSN), a massive writing survey is carried out. based totally completely on the writing survey, ZigBee is picked to accumulate a clever a ways flung sensor put together for workplacewireless robotization and domestic computerization through the idea of net of factors (IoT) [1]. furthermore, a profound exam has been reviewed on check famous deviation to find out the want of each hub. on this examination, the ZigBee gadget is constructed up and synchronization is completed relying on the character hub want and hub need is allotted for the exam of ZigBee half breed course of motion. due to this, we use ZigBee wireless topology in tree topology for reliable hub execution. We employ 3 device situations and hire three distinct parcel duration for singular conditions. in which the parcel length is $512 \mathrm{~kb}, 1024 \mathrm{~kb}$, and $2048 \mathrm{~kb}$ for Poisson, Uniform and, chi-rectangular one after the other [9].

A ZigBee upheld hobby tool were anticipated to plot and research precise system version. A profound examination has been done into singular hub need, and speciwiwireless machine duplicate devices, as an instance, community Simulator 2 (NS2), network Simulator three (NS3), OMNET++, Riverbed, and MATLAB, which backing ZigBee WSN development highlights [1]. In view of the research, Riverbed is picked as a test machine for its smooth to use interface, snappy effects and its massive scope of acknowledgment which will form and actualize ZigBee set up. furthermore, on this diploma the loads of handy sensor hubs can carry out.

From the various device convention layers of ZigBee WSN, as an example, MAC layer, installation layer, and bodily layer the recreated information are accrued because it need to be. The severa state of affairs of ZigBee device collect first rate accumulation of facts. The reenacted statistics are dissected. At that thing suitable diagrams are plotted. At ultimate, wi-amazing of issuer (QoS) of the set up ZigBee machine has been investigated with wonderful QoS parameterrs like surrender-to-end delays, statistics dropped,
Media get right of access to put off (MAC), data traffic had been given, information wi-fic sent, and international throughput and so on.

\section{MODELING AND SIMULATION}

in this phase a brief outline of the structured ZigBee tool and its setup is portrayed. the instance favored deviation situation is implemented for the want wi-figuring of each exceptional person hubs. At that factor a similar exam has been wi-finished for 3 wi-fic conditions as a protracted way as package deal appropriation strategies: Poisson, Uniform and Chi-square ability. This delineates a green approach for achieving higher wiwireless of provider (QoS).Table I illustrates an overview of the design parameters of the network.

Table:1 Overview of the network scenario

\begin{tabular}{|c|c|}
\hline Network Parameters & Status/Description \\
\hline Acknowledgment Status & Enabled \\
\hline $\begin{array}{c}\text { Acknowledgment Wait } \\
\text { Duration }\end{array}$ & $0.05 \mathrm{sec}$ \\
\hline $\begin{array}{c}\text { Number of Retransmissions } \\
\text { Channel Sensing Duration }\end{array}$ & 5 \\
\hline Data Packet Interval Time & $\begin{array}{c}\text { Poisson, Uniform, } \\
\text { Chi-square }\end{array}$ \\
\hline Data Rate & Auto Calculate \\
\hline "Utility" & Pricing \\
\hline Traffic Marking Probability & Enabled \\
\hline Data Traffic Destination & All Nodes \\
\hline Routing Strategy & Tree Routing \\
\hline Number of Nodes (overall) & 36 \\
\hline Number of Coordinator & 1 \\
\hline Number of Routers & 6 \\
\hline $\begin{array}{c}\text { Number of End Nodes } \\
\text { (Mobile and Fixed) }\end{array}$ & 29 \\
\hline Network Field & $300 \mathrm{~m} \times 300 \mathrm{~m}$ \\
\hline Duration of Simulation & $1800 \mathrm{sec}$ \\
\hline
\end{tabular}




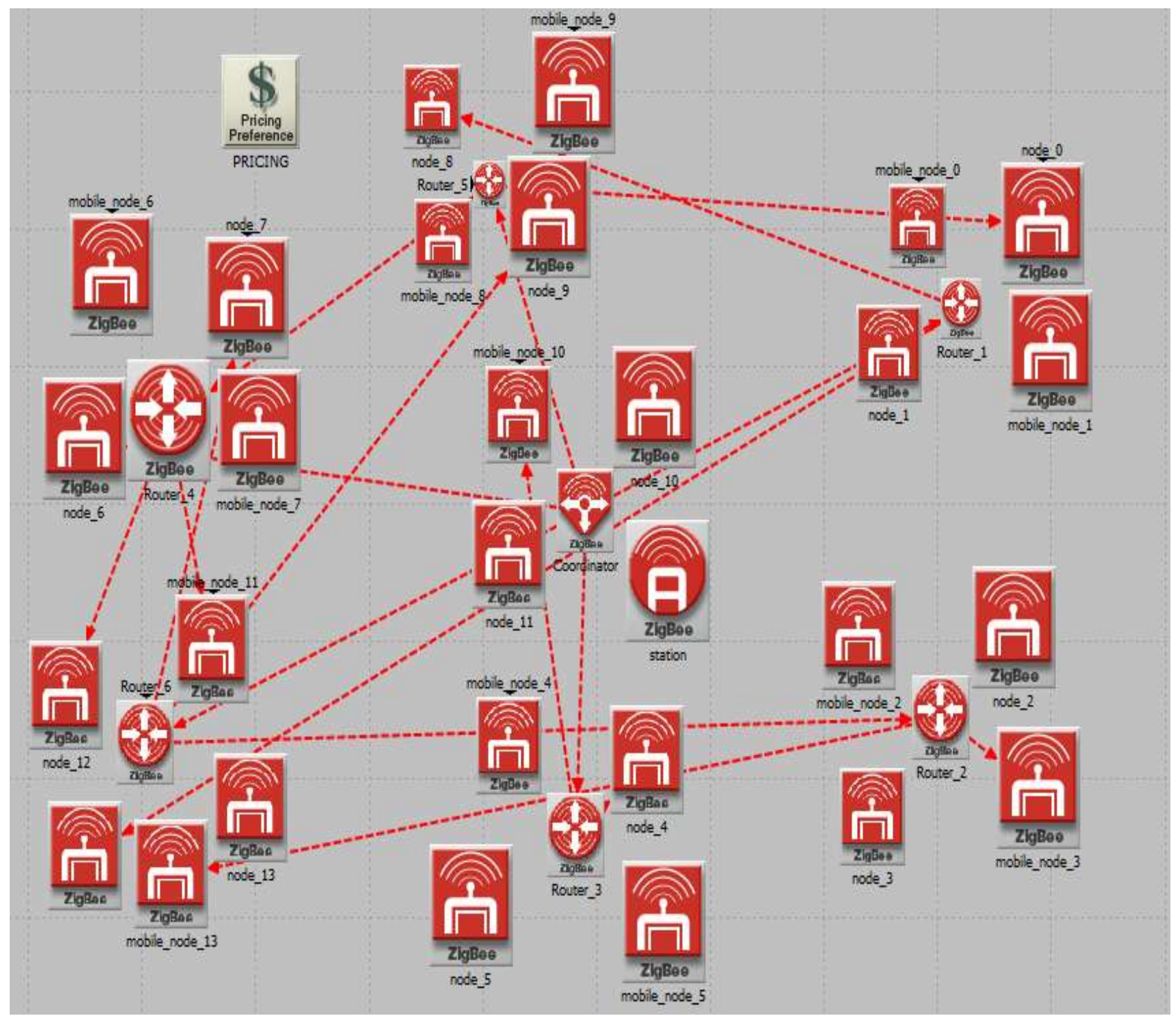

Fig. 1. The designed hybrid ZigBee network layout for simulation.

\section{A. ZigBee Priority Implementation}

A ZigBee Coordinator is a completely useful gadget for executing a ZigBee arrange. End gadgets and switches can speak with ZigBee organizer. All gadgets have a separation whether it is versatile or fixed. As indicated by the separation ZigBee system is characterized with various zone factors. The territory factor is remaining in a zone and every hub has one ZigBee switch [2]. Each hub has been set of need an incentive for recognizing its request for information transmission. Therefor need esteems is set for play out a few undertaking in a ZigBee arrange. First we expect that every hub (ZigBee end gadget) has its very own need and evaluating frameworks (values). From that point forward, the ZigBee system has been partitioned into certain zones and set the hubs to each zonal base inclination. After that we, give the need in various individual hubs in a ZigBee remote sensor arrange (WSN) and discovered QoS parameters (End gadget). From the Table II it tends to be seen that the organizer has the most elevated need esteem and the node_30 has the least need esteem.
Table:2 Proposed node payment scheme

\begin{tabular}{|c|c|c|c|}
\hline Node Name & Priority & Pricing & $\begin{array}{c}\text { Available } \\
\text { node }\end{array}$ \\
\hline Coordinator & 1 & 3300 & 1 \\
\hline Station & 2 & 3200 & 2 \\
\hline Node_10 & 3 & 3100 & 3 \\
\hline Mobile_Node_10 & 4 & 3000 & 4 \\
\hline Node_1 & 5 & 2900 & 5 \\
\hline. &. &. &. \\
\hline. &. &. &. \\
\hline . &. &. & . \\
\hline Mobiel_Node_8 & 30 & 500 & 30 \\
\hline
\end{tabular}

\section{B. Tree Routing Implementation}

The ideal steering way will be given by the ZigBee is tree directing convention by utilizing steering between arbitrary source to goal in a couple. The tree steering procedure is basically utilized in a system, when a hub begins it just knows the transitional hub and the expense to arrive at the goal [10]. In tree directing topology in the event that one hub is

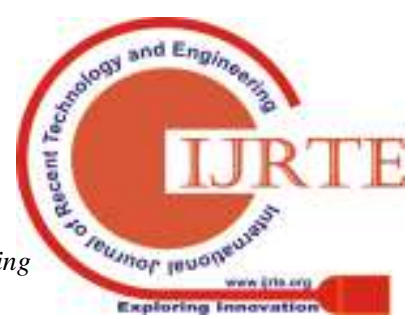




\section{QUALITY OF SERVICE (QOS) OF A ZIGBEE NETWORK THROUGH DEVICE PRIORITY WITH PACKET DISTRIBUTION TECHNIQUE}

disengaged it make the new way and interface with different hubs arrived at the goal. Countless offspring of any guardians has $(\mathrm{Cm})$ and an enormous number of the switch has $(\mathrm{Rm})$, and furthermore the profundity in the crossing tree is $\mathrm{Lm}$. The sub-squares and profundity are indicated by $\mathrm{P}$ and Cskip $(\mathrm{P})$ can be registered by the accompanying condition [10].

$\operatorname{Cskip}(\mathrm{p})=\left\{\begin{array}{l}1+C m(L m-P-1), R m=1 \\ \frac{1+C m-R m-C m R m^{L m-p-1}}{1-(R m)}, R m>1\end{array}\right\}$

Parent Cskip(P) value is always greater than the child nodes values. Also, router $\left(\mathrm{R}_{\mathrm{i}}\right)$ and child $(\mathrm{Cm})$ as well as $\mathrm{N}$-th end device child with a depth $\mathrm{d}+1$ is allocated in a regular series, using the following equations [9].

$$
\begin{gathered}
A_{(R m)}=A_{\text {Parent }}+C_{\text {skip }}(P)\left(R_{i}-1\right)+1 \\
A_{N}=A_{\text {parent }}+C_{\text {skip }}(P) R_{m}+n
\end{gathered}
$$

Where, $R_{i}$ is the router varies from 1 to $R m$ and $N$ varies from $\mathrm{Cm}-\mathrm{Rm}$ values. Every ZigBee tree routing (ZTR) node has its destination nodes that is intermediate nodes large number of hop and MAC address, Local Area Network (LAN) [9].

The relationship in ZigBee tree routing (ZTR) is a source intermediate node and a parent are as:

$$
\boldsymbol{C}_{\text {skip }}(\boldsymbol{P})>\boldsymbol{R}_{\boldsymbol{m}}>\boldsymbol{C}_{\boldsymbol{m}}
$$

\section{Priority Calculation.}

Priority calculation in network and communication is the process to calculate the order of computing devices in terms of data transmission to achieve better performance. When the individual node (devices) try to communicate with coordinator then the priority resolution is needed. Then Priority generalization is done for every node. In this research we utilized the node priority approach forming with tree routing techniques. In order to calculate the individual device priority standard deviation formula is used [11].

The summation of all device priorities in each individual node of the network is:

$\mathrm{S}_{1}=\sum_{1}^{30} D p=3300$

Average Mean, $\mathrm{X}_{1}=\frac{s_{1}}{X_{1}}$,

$\mathrm{X}_{1}=110$.

Where, the total number of element Nodes (devices) is denoted by $\mathrm{N}$. Observations are $x_{1}, x_{2}, \ldots \ldots, x_{N}$, for a numeric attribute for $\mathrm{X}$ observation.

The standard population deviation with priority values,

$$
\sigma=\sqrt{\frac{1}{N} \sum_{i=1}^{N}\left(x_{i}-\mu\right)^{2}}
$$

Here $x_{i}$, represent the total number of individual nodes mean values [1].

The distance between individual nodes is calculated in the following,

$\mathrm{D}_{1}=\mathrm{S}_{1}-$ Node_1 priority value. $\mathrm{D}_{2}=\mathrm{S}_{1}-$ Node_2 priority value. $D_{3}=S_{1}-$ Node_3 priority value............... $D_{30}=$ $\mathrm{S}_{1}-$ Node_30 priority value.

The distances of each node are,

$\mathrm{D}_{1}=(3300-500)=2800, \mathrm{D}_{2}=2700, \mathrm{D}_{3}=2600, \mathrm{D}_{4}=2500$, $\mathrm{D}_{5}=2400, \quad \mathrm{D}_{6}=2300, \quad \mathrm{D}_{7}=2200, \quad \mathrm{D}_{8}=2100, \quad \mathrm{D}_{9}=2000 \ldots$ $\mathrm{D}_{30}=500$, There may be a setting clean that the hub in which we have have been given given maximum extended need indicates higher execution. Rests of the hubs need are decided with a similar method.
D.statement (with-want ZigBee devices)

in this notion, a lower need based certainly hub (mobile_node _9) and a better want based completely hub (node_10) gadgets were taken into consideration from the tree set up topology. important dreams of a ZigBee hub (mobile_node_9) and every different hub (node_10) are given need making use of the estimating capacities. within the wake of processing, we positioned the need esteems are 1.82 and 15.21 for hub (node_10) and hub (mobile_node_9), one after the opposite. sooner or later, hub 10 receives the higher need and parade first for this situation. The hub (node_10) is near at the organizer this is the motive this hub works pretty brief.

except, the want based definitely really situation has been evaluated for the hub (mobile_node_10) and hub (node_6) from an trade scenario. The underlying dreams for hub (mobile_node_10) and hub (node_6) successively. After calculation, the want in this newsletter has been placed at 2.thirteen and eleven.eleven for the hub (mobile_node_10) and hub (node_3), for my part. Hub (mobile_node_10) is taken into consideration with better need, and the hub (node_3) is taken into consideration with decrease need esteem. The better need designates the hub to get advantage for the number one making prepared contrasted with the ZigBee Coordinator. for that reason we affirm the the rest of the hub within the need (table II)..

\section{IV.SIMULATION EFFECTS AND EVALUATION \& RESULTS}

This area is sorted out with an itemized depiction of the exam of the received endeavor consequences at the primarily based ZigBee set up execution. The principle focuse of this newsletter to investigations the man or woman hub need with-want esteems the usage of desk II. The reproduction result has been received depending on ZigBee's with need (software program and MAC) layer achievement examination. The charts are plotted subsequent to breaking down severa QoS parameters (i.E., facts dropped, statistics net web page traffic got, information net website traffic sent, give up-to-give up postponement, and Throughput, within the software layer, MAC burden, and MAC eliminate in MAC layer).

\section{A. end-To-forestall delay}

start to finish cast off is a noteworthy parameter that could effect customer's fulfillment with the gadget utility. start to complete take away alludes to the time taken for a parcel to transmit the over a tool from sender to collector.

The postponement may be decided through the accompanying circumstance [4]: $D_{\text {end-to -end }}=\left[D_{\text {trans }}+D_{\text {prop }}\right.$ $\left.+D_{\text {proc }}\right]$

Where,

$\mathrm{D}_{\text {end-end }}=$ End - to - End Delay

$\mathrm{D}_{\text {Trans }}=$ Transmission Delay

$\mathrm{D}_{\text {prop }}=$ Propagation Delay

$\mathrm{D}_{\text {proc }}=$ Processing Delay

$\mathrm{N}=$ Number of links (overall network).

Each router will have its own Transmission delay, 


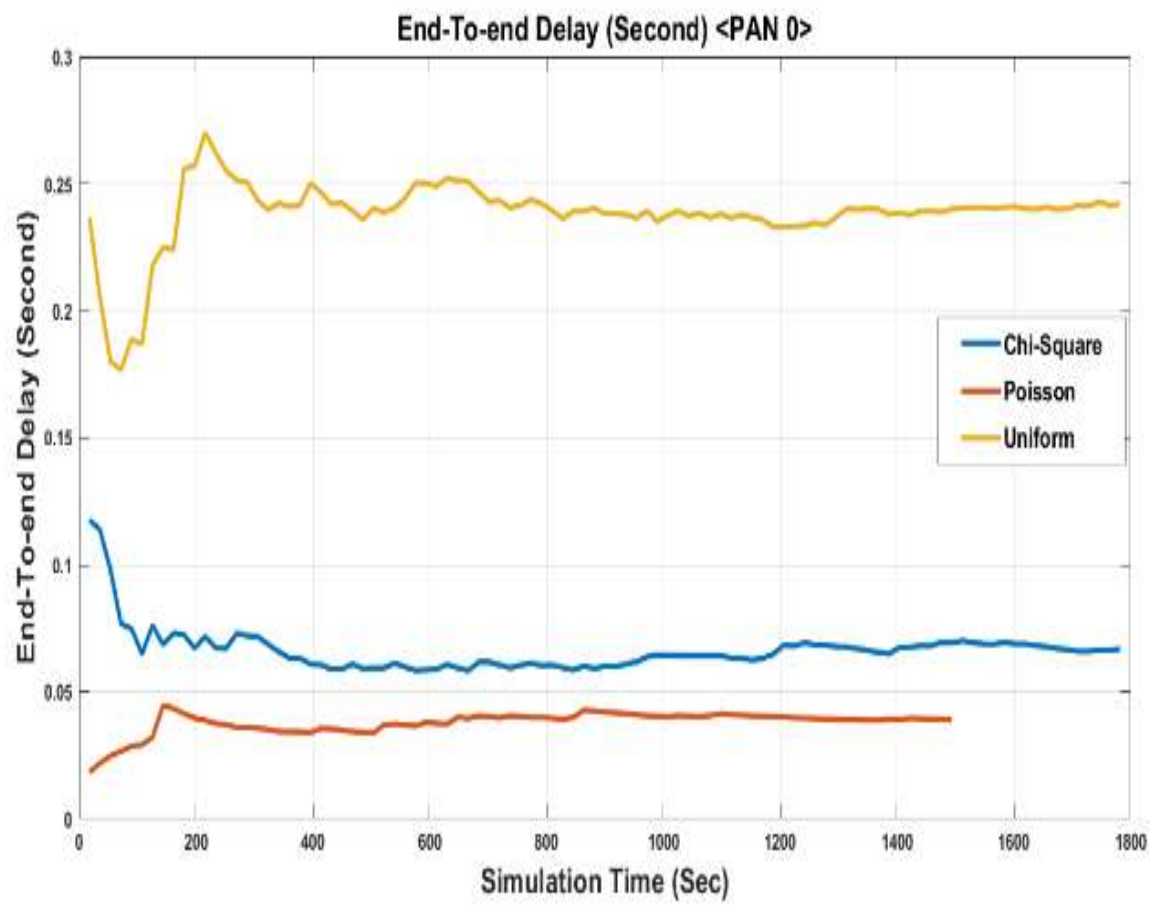

Fig. 2. End-To-end Delay With Priority Value

\section{B. Data Traffic Sent}

Data traffic is the number of data sent traffic count to move from one source to destination. Network traffic is the main component for network traffic measurement, network traffic control and network simulation results. Fig. 3 illustrates data traffic (bits/second) sent from source to destination for the 3 scenarios.

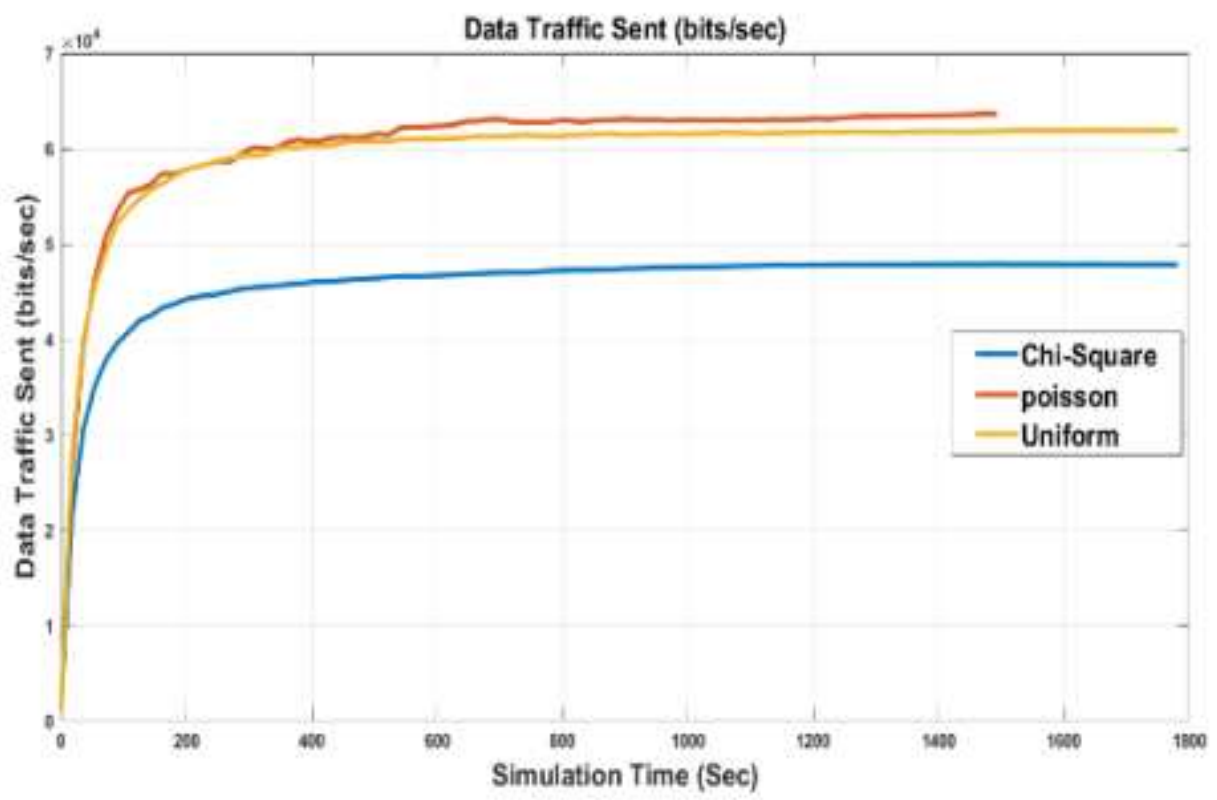

Fig. 3. Data Traffic Sent With Priority Value

\section{Data Traffic Rcvd}

Data Traffic Received (DTR) in bits per sec (bits/sec) is defined as the average number of packets per second forwarded to FTP applications by the transport layer in the network. The overall results of data traffic received for the three scenarios have been illustrated in the Fig 4 


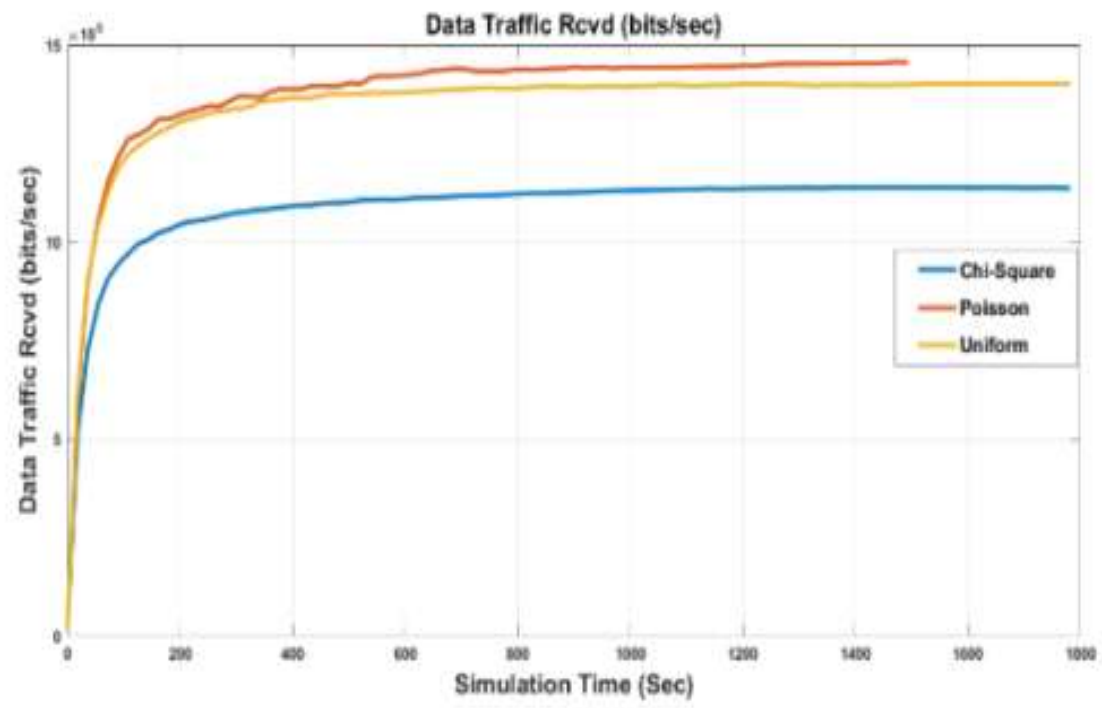

Fig. 4. Data Traffic Revd With Priority Value.

\section{Data Dropped}

Data dropped are as packet loss which is occurred when data (packets) are transferred from one destination to their target destination. Data dropped are occurred into network layer when the network is fallen down. This network layer also response for overall data dropped or packet loss. In the Fig. 5 it has been depicted that Chi-square distribution is responsible for high data dropped.

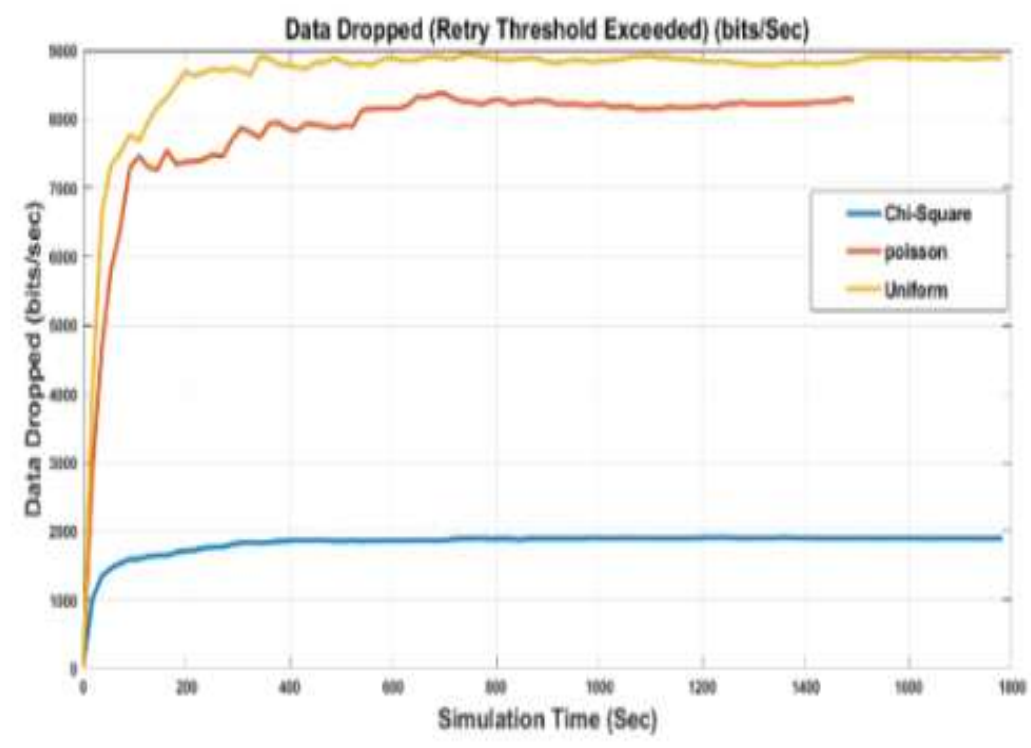

Fig. 5. Data Dropped With Priority Value.

information dropped are low while the bundle length is additionally low. Low statistics dropped gave the higher nature of administration (QoS). This exploration consists with 3 (Chi-square, Poisson, Uniform) various bundle length in three precise situations. information dropped or bundle conveyance apportion are effective execution in low parcel length and this bundle size envisioned into in keeping with unit of a duration.

\section{E. Media get right of entry to put off}

The Media access postpone is estimated as the time from whilst the data comes into the MAC layer of the ZigBee organize till it is transmitted out at the far off medium. combination sum of start to complete delay for all facts parcels. Media get admission to delay (MAC) has been portrayed in the Fig.6. For this example, the given need estimations all matters taken into consideration (Uniform, Poisson, chi-Square) are $0.002 \mathrm{sec}, 0.038 \mathrm{sec}, 0.004 \mathrm{sec}$, approximately. 


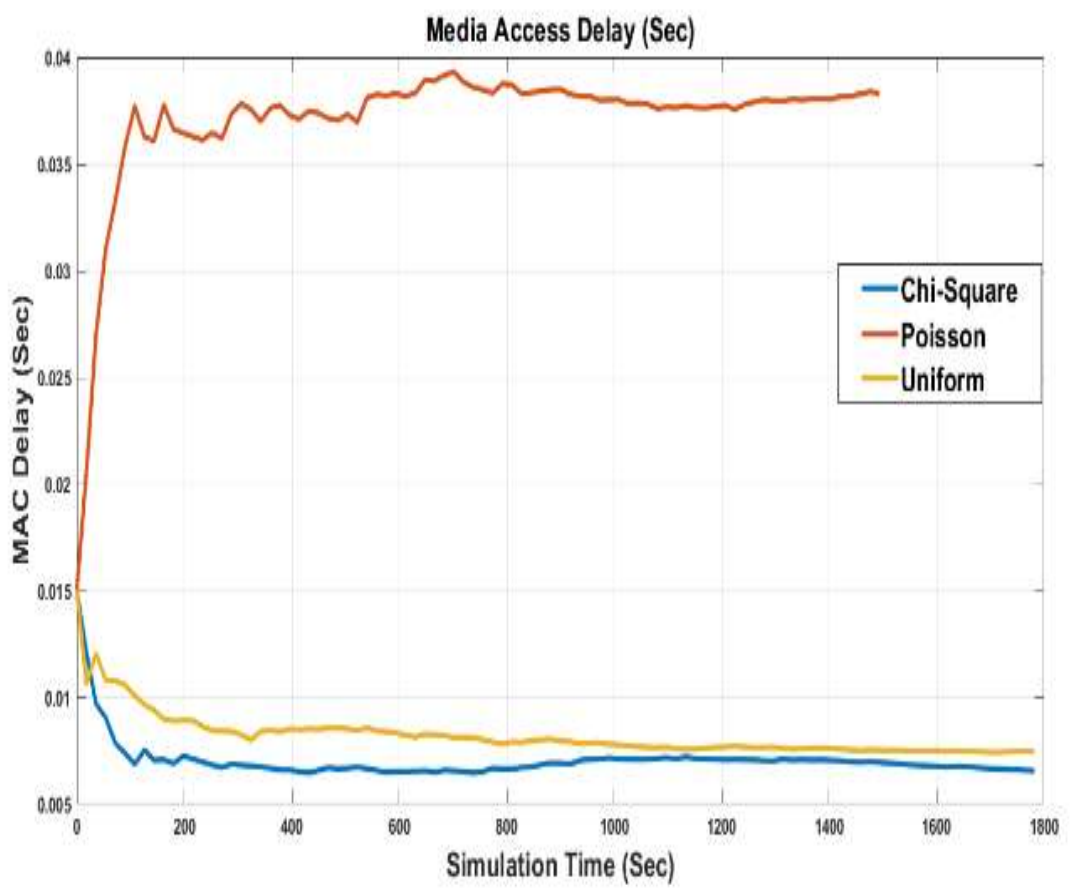

Fig. 6. Media Access Delay With Priority Value.

\section{F. Throughput}

The overall throughput of the designed ZigBee network is measured by total number of bits forwarded from ZigBee/802.15.4 MAC layer to upper layers of the ZigBee nodes. Higher throughput is always expected in any ZigBee network protocol. The communication network through successful rate from the communication network.
Throughput in a Coordinator is always higher than any nodes. In this network, throughput for the adapted three with-priority scenarios are 50,000 bits/sec, 250,000 bits/sec and 290,000 bits/sec was found for Chi-Square, Poisson, and Uniform accordingly. It has been found from the Fig 7 that Poisson distribution is responsible for higher throughput.

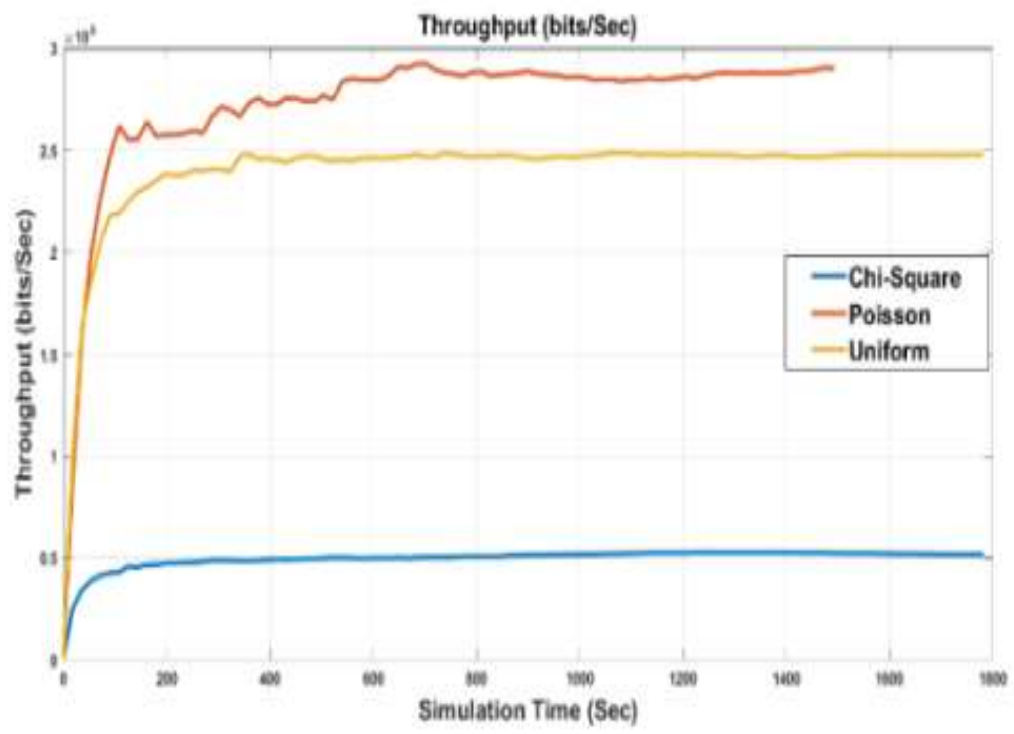

Fig. 7. Throughput With Priority Value.

Therefor we found that priority base network gives high throughput comparing without-priority network.

\section{END}

This paper speaks to the quality of provider (qos) of quite a few zigbee hubs with an accentuation on the want of numerous character hubs. After examination most people of the character hub want this newsletter arrives at the willpower that a faraway sensor coordinate with-want state of affairs offers a superior nature of management than a with out-want scenario. The overall system is synchronized

through giving want in a skilled manner. In above situations of the tool reveals that the chi-square dissemination is higher regarding facts dropped fee. However concerning throughput and give up-to-forestall eliminate poisson dissemination suggests better execution.

In future, we're able to take a look at the conduct of hard work topology taking terrific directing conference for this $1 / 2$ breed put together. 


\section{REFERENCES}

1. M. J. H. Biddut, n. Islam, m. M. Karim, and m. B. A. Miah, "an examination of qos in zigbee tool depending on veered off hub need," mag of electrical and pc engineering, vol. 2016, p. 3, 2016.

2. M. J. H. Biddut, n. Islam, m. F. H. Arif, and m. S. Rahman, "at the research of red calculation in zigbee arrange for line the executives," in informatics, electronics and imaginative and prescient (iciev), 2016 fifth worldwide convention on, 2016, pp. 408-412.

3. N. Islam, m. J. H. Biddut, a. I. Swapna, and m. H. R. Jany, "an exam on need primarily based zigbee set up execution investigation with tree steering technique," mag of computer and communications, vol. 3, no. 08, p. 1, 2015.

4. J. H. Biddut, n. Islam, m. H. R. Jany, and a. I. Swapna, "execution research of massive scale zigbee device plan via geometric form," in lawsuits of the second international convention on electric statistics and conversation technologies (eict'15), 2015, pp. 263-268.

5. N. Islam, m. J. H. Biddut, a. I. Swapna, and m. M. Rahman, "a systematic studies to restriction heap of zigbee gadget counting on actual showing," in electric and pc engineering (wiecon-ece), 2015 ieee global wie conference on, 2015, pp. 139-142.

6. N. Islam, m. J. H. Biddut, a. I. Swapna, and s. Asaduzzaman, "superior fantastic of provider in zigbee coordinate with actual showing," in advances in electric powered powered engineering (icaee), 2015 global convention on, 2015, pp. 174-177.

7. "zigbee alliance," zigbee alliance. [online]. Available: https://www.zigbee.org/. [accessed: 14-nov-2018].

8. N. Islam, c. C. Bawn, j. Hasan, a. I. Swapna, and m. S. Rahman, "nature of management exam of ethernet tool relying on package length," journal of laptop and communications, vol. Four, no. 04, p. Sixty three, 2016.

9. M. J. H. Biddut, m. F. H. Arif, and n. Islam, "line the board of purple empowered zigbee device relying on parcel length kinds and circulate strategies," in 2017 worldwide conference on electric, laptop and communique engineering (ecce), cox's bazar, bangladesh, 2017, pp. 798-802.

10. P. S. Kumar and a. R. Babu, "a survey on routing techniques in zigbee wi-fi networks in assessment with one-of-a-kind wireless networks," indian Journal of era and generation, vol. 10, no. 40 two, 2018.

11. J. Han, m. Kamber, and j. Pei, records mining: requirements and techniques, elsevier, massive apple, large apple, united states of america, 2011. 